\title{
Correlation Analysis of Fish-eye Lens Camera for Acquiring Reliable Orientation Parameters
}

\author{
Kang Hyeok Choi, ${ }^{1}$ Yongmin $\mathrm{Kim},{ }^{2}$ and Changjae $\mathrm{Kim}^{1 *}$ \\ ${ }^{1}$ Department of Civil and Environmental Engineering, Myongji University, \\ 116 Myongji-ro, Cheoin-gu, Yongin, Gyeonggi-do 17058, Korea, \\ ${ }^{2}$ Korea Land and Geospatial Informatix Corporation, \\ 120 Giji-ro, Deokjin-gu, Jeonju Jeollabuk-do, 54867, Korea
}

(Received September 8, 2019; accepted November 13, 2019)

Keywords: fish-eye lens camera, perspective projection camera, reliable orientation parameters, correlation analysis, test bed shapes

The fish-eye lens camera has the advantage of efficient acquisition of image data through a wide field of view. However, unlike the perspective projection camera, a strong geometric distortion effect appears in the image. Such characteristics must be precisely analyzed through self-calibration to determine the interior characteristics of the camera. The reliability of the derived camera parameters is highly affected by the correlation between orientation parameters. However, a detailed analysis of the correlation between the orientation parameters of the fish-eye lens is insufficient. In this research, we compared the correlation tendency while considering different types of test beds and projection models. As the shape of the test bed changed, the correlation of the perspective projection and fish-eye lens cameras showed a distinctly different tendency. On the basis of the results of correlation analysis, guidelines for acquiring reliable interior orientation parameters (IOPs) and exterior orientation parameters (EOPs) of a camera were provided.

\section{Introduction}

\subsection{Background}

Owing to its characteristics, the fish-eye lens camera has been widely used for indoor and outdoor location-based services, virtual reality (VR), 3D modelling, leisure sports, and robotics. Marković et al. and Caruso et al. used a fish-eye lens camera for mobile robot implementation, and Sánchez et al. used it for urban navigation. ${ }^{(1-3)}$ Sreedhar et al. built a system for virtual reality applications using fish-eye lens cameras. ${ }^{(4)}$ Schöps et al. used such cameras to produce a three-dimensional model for a large area. ${ }^{(5)}$ Campos et al. built a backpack-mounted fisheye lens camera system and used it for forest mapping. ${ }^{(6)}$ Kühling built an advanced driving

*Corresponding author: e-mail: cjkim@mju.ac.kr https://doi.org/10.18494/SAM.2019.2609 
assistance system using multiple fish-eye lens cameras. ${ }^{(7)}$ Although a fish-eye lens camera has a wide viewing angle, it has the disadvantage of strong geometric distortion in the image. Therefore, the process of determining the interior orientation parameters (IOPs) of the camera must be implemented to correct the image distortion. ${ }^{(8,9)}$ The relevant previous studies can be categorized mostly into two trends: (1) the verification of each projection model of the fish-eye lens ${ }^{(10,11)}$ and (2) comparisons between the projection models and the possibility of substituting a projection model. ${ }^{(9,12,13)}$ Some of the previous studies found a correlation between IOPs and exterior orientation parameters (EOPs), which has rarely been analyzed in detail. ${ }^{(9,12,14)}$

The correlation between the camera's orientation parameters is a very important indicator of calibration accuracy, because it has a very large impact on the reliability of the parameters. A high correlation between the orientation parameters significantly decreases the reliability of the calibration result.

For a perspective projection model camera, a method of reducing the correlation between parameters has been proposed in various papers. ${ }^{(15-17)}$ However, a detailed analysis of the correlation between the camera orientation parameters of the fish-eye lens is insufficient. Since the fish-eye lens camera differs from the conventional camera in the projection model equation, the way in which the correlation occurs may also differ, which can have a significant impact on the parameter reliability of the fish-eye lens camera.

\subsection{Purpose and scope of research}

The purposes of this research are (1) to analyze the tendency of parameter correlation according to different camera types, test bed rotation angles, and test bed types, and (2) to provide guidelines for acquiring reliable IOPs and EOPs of a camera.

In this paper, we consider five projection models, one of which is a perspective projection model and the other four being representative projection models of the fish-eye lens camera (i.e., equidistant, equisolid-angle, orthogonal, and stereographic projection models). ${ }^{(18-21)}$ Various experiments considering different test bed rotation angles and test bed types are carried out; then, the reliability of the derived camera parameters is evaluated on the basis of the results of correlation analysis. In this research, we utilized simulated datasets while considering the systematic and stochastic models that have been well-established and verified in the photogrammetric community. At this stage, the simulation might be very useful in such situations handling numerous cases with different camera types, test bed rotation angles, and test bed types. Moreover, since the simulation is carried out after setting all the involved variables, the derived camera parameters can be directly compared and analyzed with the preset values; such comparison is almost impossible in the case of real data.

The research contents of this paper start from Sect. 2 with the description of projection and lens distortion models of fish-eye lens cameras. In Sect. 3, we explain how to design an experiment for correlation analysis. In Sect. 4, we analyze the results of correlation analysis and provide guidelines for image acquisition with low correlations. Finally, conclusions and an upcoming work are discussed in Sect. 5. 


\section{Mathematical Model of Camera}

The image recording of an optical camera can be mathematically expressed using projection and lens distortion models. ${ }^{(22)}$ The projection models can be divided into perspective and fisheye lens projection models for normal and fish-eye lens cameras, respectively. Moreover, the fish-eye lens projections can be divided into four different models (i.e., equidistant, equisolidangle, orthogonal, and stereographic projection models). The lens distortion model, which explains the distortion caused by the camera lens, is used in both the perspective projection camera and the fish-eye lens camera.

\subsection{Projection models}

The mathematical relationship between the object space and the image space can be described using the projection models as shown in Eqs. (1)-(8), ${ }^{(14)}$ where $\boldsymbol{M}$ is the rotation matrix between the ground and camera coordinate systems. $(X, Y, Z)$ are the coordinates of the object point and $\left(X_{0}, Y_{0}, Z_{0}, \omega, \varphi, \kappa\right)$ are the EOPs of a camera. $f$ is the focal length, $\theta$ is the incident angle of the object point, and $r$ is the distance from the principal point to the image point. $\left(x_{p}, y_{p}\right)$ are the coordinates of the principal point.

$$
\begin{aligned}
& {\left[\begin{array}{l}
U \\
V \\
W
\end{array}\right]=\boldsymbol{M}(\omega, \varphi, \kappa)\left[\begin{array}{c}
X-X_{0} \\
Y-Y_{0} \\
Z-Z_{0}
\end{array}\right]} \\
& \text { if Perspective Projection: } r=f \tan \theta \\
& \text { elseif Equidistant Projection: } r=f \tan \theta \\
& \text { elseif Equisolid-angle Projection: } r=f \tan \theta \\
& \text { elseif Orthogonal Projection: } r=f \tan \theta \\
& \text { elseif Stereographic Projection: } r=f \tan \theta \\
& x=x_{p}-\frac{r}{\sqrt{U^{2}+V^{2}}} U \\
& y=y_{p}-\frac{r}{\sqrt{U^{2}+V^{2}}} V
\end{aligned}
$$




\subsection{Lens distortion model}

Lens distortions are calculated using Eqs. (9)-(13), ${ }^{(23)}$ where $\Delta x$ and $\Delta y$ are the distortions of the image coordinates $x$ and $y$, respectively, and $K_{1}, K_{2}, K_{3}, P_{1}, P_{2}, A_{1}$, and $A_{2}$ are distortion parameters. $r$ in Eqs. (2)-(6) can be rewritten as the distance between the image coordinates $(x, y)$ and the principal point coordinates $\left(x_{p}, y_{p}\right)$ as described in Eq. (13). Equations (14) and (15) are the final mathematical models of an optical camera. The final coordinates of the image point are composed of projection [Eqs. (7) and (8)] and lens distortion [Eqs. (9) and (10)] models.

$$
\begin{gathered}
\Delta x=\bar{x}\left(K_{1} r^{2}+K_{2} r^{4}+K_{3} r^{6}\right)+P_{1}\left(r^{2}+2 \bar{x}^{2}\right)+2 P_{2} \bar{x} \bar{y}+A_{1} \bar{x}+A_{2} \bar{y} \\
\Delta y=\bar{y}\left(K_{1} r^{2}+K_{2} r^{4}+K_{3} r^{6}\right)+2 P_{1} \bar{x} \bar{y}+P_{2}\left(r^{2}+2 \bar{y}^{2}\right) \\
\bar{x}=x-x_{p} \\
\bar{y}=y-y_{p} \\
r=\sqrt{\bar{x}^{2}+\bar{y}^{2}} \\
x=x_{p}-\frac{r}{\sqrt{U^{2}+V^{2}}} U+\Delta x \\
y=y_{p}-\frac{r}{\sqrt{U^{2}+V^{2}}} V+\Delta y
\end{gathered}
$$

\section{Correlation Analysis Design}

Experiments were performed to determine the correlation tendency between orientation parameters according to the relative position between the camera and the test bed and the type of test bed. The correlations that were the subject of the analysis are $x_{p}-X_{0}, y_{p}-Y_{0}, x_{p}-Y_{0}$, $y_{p}-X_{0}$, and $f-Z_{0}$. The experiments were carried out in the following order. First, simulation image points were calculated using previously determined virtual IOPs, EOPs, and object points in a test bed. Next, the true values of all the variables except for the two orientation parameters (to examine the correlation) were substituted into Eqs. (14) and (15). Finally, when one value of the orientation parameter was changed, the values of the other orientation parameters satisfying Eqs. (14) and (15) were calculated, and the correlation between the two orientation parameters was calculated.

The IOPs, specifications, and EOPs of the camera used in the simulation were set as shown in Tables 1 and 2. The focal lengths were set to 2.5 and $5.0 \mathrm{~mm}, x_{p}, y_{p}$, and the distortion parameters were all set to zero, the pixel size was set to $0.0035 \mathrm{~mm}$, and the image size was set to $3000 \times 2400$ as shown in Table 1 . The values in Table 1 were based on a Sunnex DSL315 fish-eye lens camera and modified for this research. The EOPs of the camera were all set to zero, except for $\kappa$, as shown in Table 2 , and to 0 or $90^{\circ}$ for $\kappa$. 
Table 1

IOPs and specifications of camera in correlation analysis.

\begin{tabular}{|c|c|c|c|c|c|c|c|c|c|c|}
\hline \multirow{3}{*}{ IOP } & \multirow{2}{*}{$f(\mathrm{~mm})$} & \multirow{2}{*}{$x_{p}(\mathrm{~mm})$} & \multirow{2}{*}{$y_{p}(\mathrm{~mm})$} & \multicolumn{7}{|c|}{ Distortion parameter } \\
\hline & & & & $K_{1}$ & $K_{2}$ & $K_{3}$ & $P_{1}$ & $P_{2}$ & $A_{1}$ & $A_{2}$ \\
\hline & $2.5 / 5.0$ & 0 & 0 & 0 & 0 & 0 & 0 & 0 & 0 & 0 \\
\hline \multirow{3}{*}{ Camera specification } & \multirow{2}{*}{\multicolumn{3}{|c|}{ Pixel size (mm) }} & \multicolumn{7}{|c|}{ Image size (pixel) } \\
\hline & & & & & $x$ & & & & & \\
\hline & \multicolumn{3}{|c|}{0.0035} & & 3000 & & \multicolumn{4}{|c|}{2400} \\
\hline
\end{tabular}

Table 2

EOPs of camera.

\begin{tabular}{cccccc}
\hline$X_{0}(\mathrm{~m})$ & $Y_{0}(\mathrm{~m})$ & $Z_{0}(\mathrm{~m})$ & $\omega\left(^{\circ}\right)$ & $\varphi\left(^{\circ}\right)$ & $\kappa\left({ }^{\circ}\right)$ \\
\hline 0 & 0 & 0 & 0 & 0 & $0 / 90$ \\
\hline
\end{tabular}

Table 3

(Color online) Test bed configuration for plane-type test bed rotation.

\begin{tabular}{cc}
\hline $\begin{array}{c}\text { Relative location between } \\
\text { test bed and camera }\end{array}$ & $\begin{array}{c}\text { Test bed rotation angle } \\
\text { setting (top view) }\end{array}$ \\
\hline
\end{tabular}

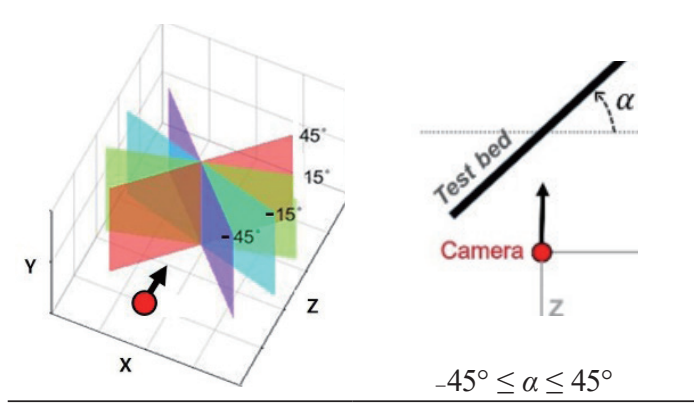

The test bed was set up by two methods: (1) plane-type test bed rotation and (2) test bed type change. First, "correlation analysis when rotating a plane-type test bed" was performed, because many conventional studies have used a plane-type test bed for camera self-calibration. ${ }^{(21,24)}$

Next, "correlation analysis when the type of test bed was changed" was performed to reflect previous studies using some types of test beds installed on artifacts such as inside or outside the walls of a building. ${ }^{(9,20)}$ In this paper, the geometry of the above-mentioned artifacts was interpreted as consisting of a plane and concave and convex corners. Therefore, three types of test beds, namely, the plane, A (concave corner), and V (convex corner) types, were used for the analysis.

Table 3 shows the test bed configuration for the plane-type test bed rotation. The red "circle arrow" in the table denotes the position and direction of the camera, and $\alpha$ is the rotation angle of the test bed. The size of the plane-type test bed was set to $4 \times 3 \mathrm{~m}^{2}$ (width $\times$ height) and $\alpha$ was changed from -45 to $45^{\circ}$ by $1^{\circ}$ intervals.

Table 4 shows the test bed settings for the test bed type change. The test bed type was adjusted by changing the angle $\beta$ between the two planes from 90 to $270^{\circ}$. The test bed was of the $\mathrm{V}$ type when $\beta$ was less than $180^{\circ}$, of the plane type when $\beta$ was $180^{\circ}$, and of the A type when $\alpha$ was greater than $180^{\circ}$. The size of the plane-type test bed was the same as that of the case of "the plane-type test bed rotation", and the A- or V-type test bed consisted of two planes set to $2 \times 3 \mathrm{~m}^{2}$. 
Table 4

(Color online) Test bed configuration for test bed type change.

Relative location
between test bed and camera

Table 5

(Color online) Test bed location setting.

\begin{tabular}{ccc}
\hline $\bar{X}(\mathrm{~m})$ & $\bar{Y}(\mathrm{~m})$ & $d=|\bar{Z}|(\mathrm{m})$ \\
\hline \multirow{2}{*}{0} & \multirow{2}{*}{0} & 2 \\
\cline { 2 - 2 } & & 3 \\
\hline
\end{tabular}

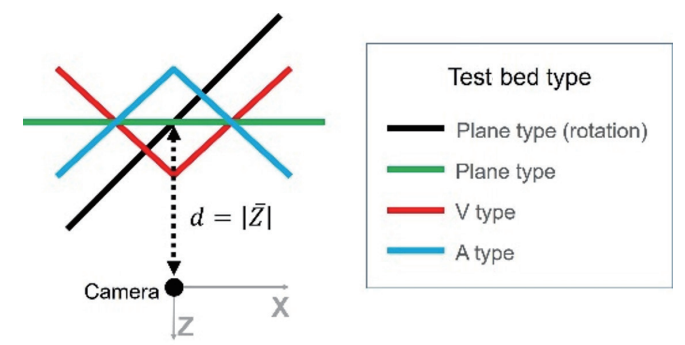

The location of the test bed was set as shown in Table 5 , where $\bar{X}, \bar{Y}$, and $\bar{Z}$ are the mean values of the coordinates $X, Y$, and $Z$ (i.e., object points) on the test bed, respectively. In this research, we set $\bar{X}$ and $\bar{Y}$, to 0 so that the camera looks at the centroid of the test bed and $d$ (the absolute value of $\bar{Z}$ ) changes to 2,3 , and $4 \mathrm{~m}$.

\section{Results of Correlation Analysis}

A comprehensive analysis of the correlations between the orientation parameters was performed on the basis of the results of various experiments. Different cases of correlation analysis between the orientation parameters were considered, as shown in Table 6, according to the combination of orientation parameters, test bed variation methods (i.e., test bed rotation, and test bed type change), and EOP $k$. Since the correlation between $f$ and $Z_{0}$ is not affected by $\kappa$, it is expressed as one case for each test bed setting method. 
Table 6

(Color online) Considered experiments for correlation analysis.

\begin{tabular}{ccccc}
\hline \multirow{2}{*}{ Orientation parameter combination } & \multicolumn{2}{c}{ Test bed rotation } & \multicolumn{2}{c}{ Test bed type change } \\
\cline { 2 - 5 } & $\kappa=0^{\circ}$ & $\kappa=90^{\circ}$ & $\kappa=0^{\circ}$ & $\kappa=90^{\circ}$ \\
\hline$x_{p}-X_{0}$ & Case 1 & Case 5 & Case 9 & Case 13 \\
$y_{p}-X_{0}$ & Case 2 & Case 6 & Case 10 & Case 14 \\
$x_{p}-Y_{0}$ & Case 3 & Case 7 & Case 11 & Case 15 \\
$y_{p}-Y_{0}$ & Case 4 & Case 8 & Case 12 & Case 16 \\
$f-Z_{0}$ & \multicolumn{3}{c}{ Case 17 } & \multicolumn{2}{c}{ Case 18 } \\
\hline
\end{tabular}

All the cases in Table 6 were divided into four categories on the basis of the similarity of the results. The two categories being highlighted in yellow and green are the cases of the test bed rotation, and the other two categories in grey and blue are the cases of the type of test bed change.

In terms of the structure of the paper, Sect. 4.1 deals with the results of correlation analyses for test bed rotations. In Sect. 4.2, we show the results of correlation analyses for test bed changes. One should also note that all the experiments described in Sects. 4.1 to 4.2 use a focal length of $2.5 \mathrm{~mm}$. The effect of focal length change on the correlation is discussed in Sect. 4.3 while comparing the results from focal lengths of 2.5 and $5.0 \mathrm{~mm}$. Finally, in Sect. 4.4, we discuss the results of comprehensive analysis on the basis of the findings described in Sects. 4.1 to 4.3 .

\subsection{Correlation for test bed rotation}

In this section, we show how the correlations between orientation parameters behaved when the relative location between the camera and the test bed changed. Moreover, the correlation differences between the perspective and fish-eye lens projections were confirmed. The results of correlation analyses for the categories in yellow (as seen in Table 6) are shown in Figs. 1 and 2. On the other hand, those of correlation analyses for the categories in green are shown in Figs. 3 and 4.

More specifically, Fig. 1 shows the correlations of $x_{p}-X_{0}\left(\kappa=0^{\circ}\right), y_{p}-Y_{0}\left(\kappa=0^{\circ}\right), y_{p}-X_{0}(\kappa=$ $\left.90^{\circ}\right), x_{p}-Y_{0}\left(\kappa=90^{\circ}\right)$, and $f-Z_{0}$ (Cases $1,4,6,7$, and 17 in Table 6). Regardless of the projection models, the similarities in the correlation changes observed in the figure were as follows. First, the correlation between IOPs and EOPs is highest when the camera looks at the test bed orthogonally (when $\alpha=0^{\circ}$ ), and the correlation decreases when the camera looks at the test bed obliquely. Next, the correlation between the orientation parameters shows a lower value as the distance $(d)$ between the test bed and the camera decreases.

The characteristics of the correlation tendency according to the projection model are as follows. First, the fish-eye lens camera, compared with the perspective projection camera, has a smaller change in correlation coefficient when the camera looks at the test bed obliquely. Next, the level of correlation change is in the order of perspective, stereographic, equidistant, equisolid-angle, and orthogonal projections. 


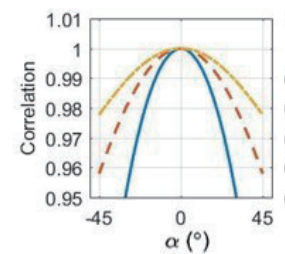

(a)
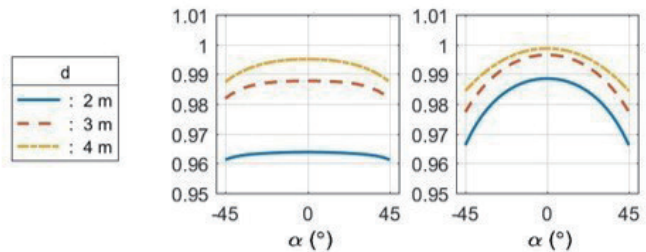

(d)

(e)

Fig. 1. (Color online) Correlations between orientation parameters for test bed rotation (Cases 1, 4, 6,7 , and 17; $2.5 \mathrm{~mm}$ focal length): (a) perspective, (b) equidistant, (c) equisolid-angle, (d) orthogonal, and (e) stereographic projection models.

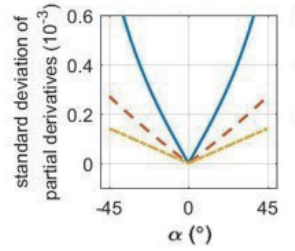

(a)

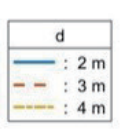

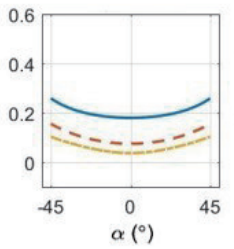

(b)

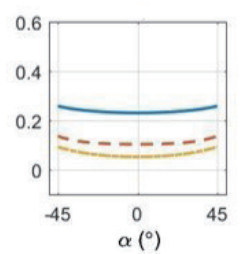

(d)

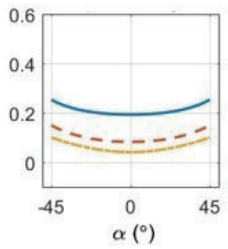

(c)

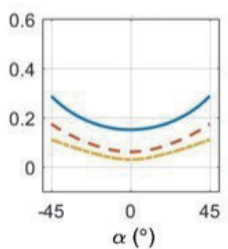

(e)
Fig. 2. (Color online) Standard deviations of partial derivatives for test bed rotation (Cases 1, 4, 6, 7, and $17 ; 2.5 \mathrm{~mm}$ focal length): (a) perspective, (b) equidistant, (c) equisolid-angle, (d) orthogonal, and (e) stereographic projection models.

Figure 2 shows the standard deviations of partial derivatives of mathematical models (i.e., Eqs. 14 and 15) in Cases 1, 4, 6, 7, and 17. The partial derivative shows the strength of the linear relationship between the parameters in question. As long as we deal with all the object points shown in an image, the dispersion of partial derivatives (i.e., standard deviation of partial derivatives) should be considered instead of one partial derivative. The low standard deviation of partial derivatives leads to a strong correlation between the parameters in question. Also, vice versa, a high standard deviation leads to a weak correlation between the parameters. For example, the high standard deviation of partial derivatives means that the partial derivatives for all the points in an image are mostly different from each other; hence, the two orientation parameters in question do not have a strong linear relationship (i.e., correlation). Figures 1 and 2 show such an inversely proportional relationship.

Figure 3 shows the correlations of $x_{p}-Y_{0}\left(\kappa=0^{\circ}\right), y_{p}-X_{0}\left(\kappa=0^{\circ}\right), x_{p}-X_{0}\left(\kappa=90^{\circ}\right)$, and $y_{p}-$ $Y_{0}\left(\kappa=90^{\circ}\right)($ Cases 2, 3, 5, and 8 in Table 6) for test bed rotation. As can be seen in the figure, all the correlation values for five different projection models are very close to zero. This can be explained from the fact that the corresponding partial derivative is zero or very close to zero (Fig. 4), which means that the relevant parameters do not affect each other.

\subsection{Correlation for test bed type change}

The correlations between orientation parameters were analyzed while changing the test bed type in this section. The perspective and fish-eye lens projection models showed a significantly different correlation tendency compared with the cases of test bed rotation.

The experimental results for the first category including Cases 9, 12, 14, 15, and 18 in Table 6 are shown in Figs. 5 and 6. Figure 5 shows the correlations of $x_{p}-X_{0}\left(\kappa=0^{\circ}\right), y_{p}-Y_{0}$ 


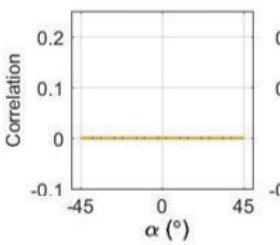

(a)

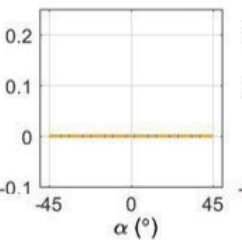

(b)

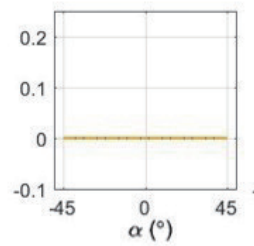

(d)

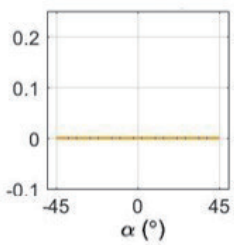

(c)

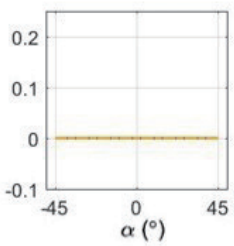

(e)

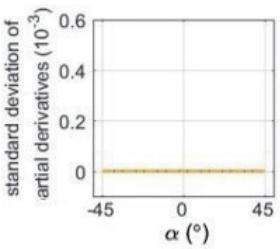

(a)

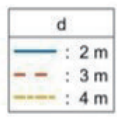

Fig. 3. (Color online) Correlations between orientation parameters for test bed rotation (Cases 2, 3,5 , and $8 ; 2.5 \mathrm{~mm}$ focal length): (a) perspective, (b) equidistant, (c) equisolid-angle, (d) orthogonal, and (e) stereographic projection models. projection models.

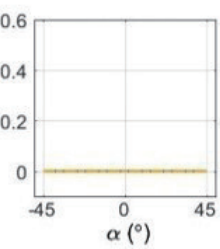

(b)

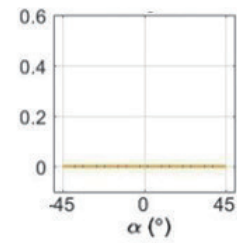

(d)

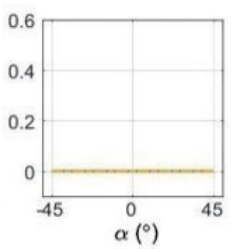

(c)

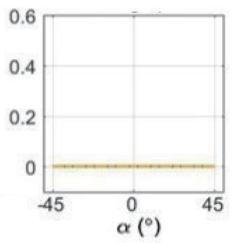

(e)

Fig. 4. (Color online) Standard deviations of partial derivatives for test bed rotation (Cases 2, 3, 5, and 8; 2.5 $\mathrm{mm}$ focal length): (a) perspective, (b) equidistant, (c) equisolid-angle, (d) orthogonal, and (e) stereographic

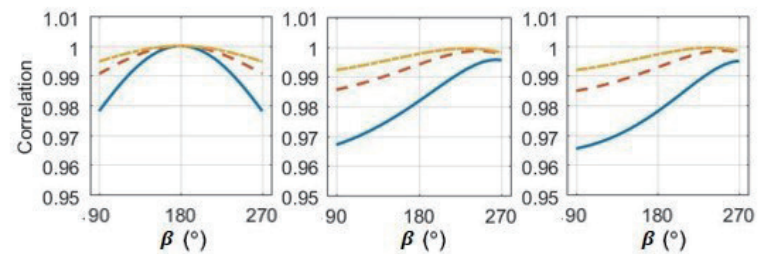

(a)

(b)

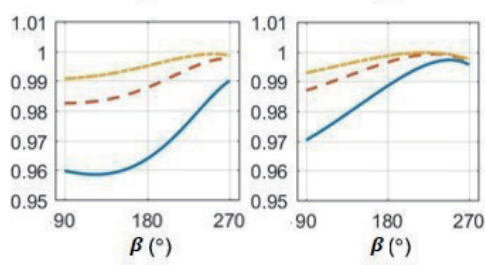

(d)

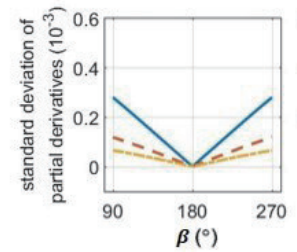

(a)

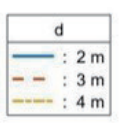

(e)

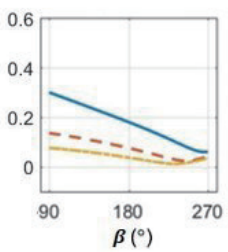

(b)

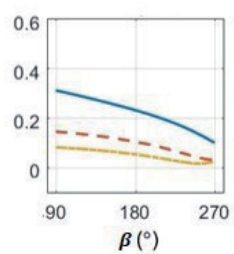

(d)

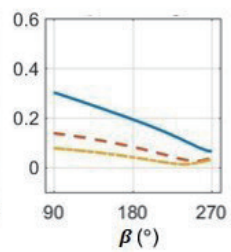

(c)

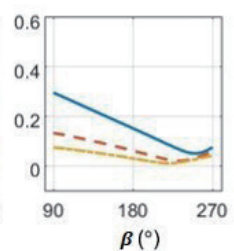

(e)

Fig. 5. (Color online) Correlations between orientation parameters for test bed type change (Cases 9, 12, 14, 15, and 18; $2.5 \mathrm{~mm}$ focal length): (a) perspective, (b) equidistant, (c) equisolid-angle, (d) orthogonal, and (e) stereographic projection models.

Fig. 6. (Color online) Standard deviations of partial derivatives for test bed type change (Cases 9, 12, 14, 15, and 18; $2.5 \mathrm{~mm}$ focal length): (a) perspective, (b) equidistant, (c) equisolid-angle, (d) orthogonal, and (e) stereographic projection models.

$\left(\kappa=0^{\circ}\right), y_{p}-X_{0}\left(\kappa=90^{\circ}\right), x_{p}-Y_{0}\left(\kappa=90^{\circ}\right)$, and $f-Z_{0}$. The $x$-axis indicates the angle between the two planes constituting the test bed (as shown in Table 4), and the $y$-axis indicates the correlation between the orientation parameters. The most notable feature shown in Fig. 5 is that the correlation tendencies of the perspective and fish-eye lens projections are significantly different. Recall that this research considers three different types of test beds: $\mathrm{V}\left(90^{\circ} \leq \beta<\right.$ $\left.180^{\circ}\right)$, plane $\left(\beta=180^{\circ}\right)$, and $\mathrm{A}\left(180^{\circ}<\beta \leq 270^{\circ}\right)$ types. The perspective projection showed the 
highest correlation in the plane-type test bed and similar correlations in the A- and V-type test beds. On the other hand, the fish-eye lens projection generally showed a higher correlation in the order test bed of V-, plane-, and A-type test beds. As the distance d between the test bed and the camera increases, the correlation between the orientation parameters tends to increase. Also, the larger the standard deviation of the partial derivatives, the lower the correlation (Fig. 6).

The second category, including Cases 10,11, 13, and 16, showed correlation values close to zero (similarly to Fig. 3). The standard deviations of partial derivatives for the second category were also very close to zero (similarly to Fig. 4). Hence, the figures in these cases were not included in this paper. Such tendency means that there is no correlation between the relevant orientation parameters.

\subsection{Effect of focal length change on the correlation}

In this section, additional experiments with a focal length of $5.0 \mathrm{~mm}$ were carried out and the results of these experiments were compared with those shown in Sects. 4.1 and 4.2. The results of the analyses of the cases in yellow (Cases 1, 4, 6, 7, and 17) and grey (Cases 9, 12, 14, 15, and 18) are shown in Figs. 7 and 8, respectively. Figure 7 shows the correlations of $x_{p}-X_{0}(\kappa$ $\left.=0^{\circ}\right), y_{p}-Y_{0}\left(\kappa=0^{\circ}\right), y_{p}-X_{0}\left(\kappa=90^{\circ}\right), x_{p}-Y_{0}\left(\kappa=90^{\circ}\right)$, and $f-Z_{0}$ (Cases 1, 4, 6, 7, and 17 in Table 6). Figure 8 shows the correlations of $x_{p}-X_{0}\left(\kappa=0^{\circ}\right), y_{p}-Y_{0}\left(\kappa=0^{\circ}\right), y_{p}-X_{0}\left(\kappa=90^{\circ}\right), x_{p}-Y_{0}(\kappa=$ $90^{\circ}$ ), and $f-Z_{0}$ (Cases 9, 12, 14, 15, and 18 in Table 6). By comparing Figs. 1 and 7, and Figs. 3 and 8 , we confirmed that the overall tendencies of the correlations do not significantly change. Hence, the focal length change does not affect the correlation of camera orientation parameters.

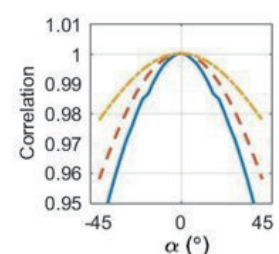

(a)

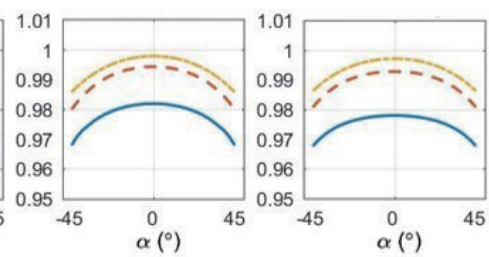

(b)
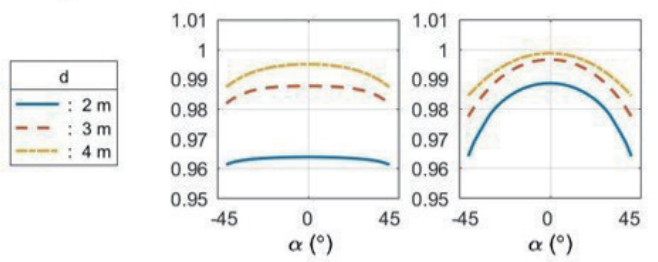

(d) (c)

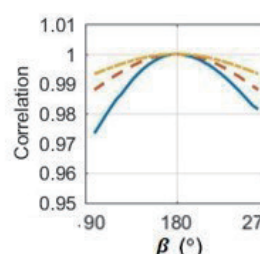

(a)

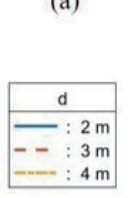

(e)
Fig. 7. (Color online) Correlations between orientation parameters for test bed rotation (Cases 1, 4, 6,7 , and 17; $5.0 \mathrm{~mm}$ focal length): (a) perspective, (b) equidistant, (c) equisolid-angle, (d) orthogonal, and (e) stereographic projection models.
Fig. 8. (Color online) Correlations between orientation parameters for test bed type change (Cases 9, 12, 14, 15, and 18; $5.0 \mathrm{~mm}$ focal length): (a) perspective, (b) equidistant, (c) equisolid-angle, (d) orthogonal, and (e) stereographic projection models. 
The correlations of the green (Cases 2, 3, 5, and 8) and blue (Cases 10, 11, 13, and 16) cases were very close to zero, the same as that observed in Figs. 3 and 4 shown in Sect. 4.1. In other words, we do not see any correlation between the relevant orientation parameters.

\subsection{Comprehensive analysis of correlation between orientation parameters}

The correlation analysis could be divided into two parts: (i) analysis supporting the results of previous studies (based on a perspective projection camera), and (ii) correlation characteristics between orientation parameters observed through this research.

First, the correlation analysis supporting the results of existing studies is as follows.

1. When using a plane-type test bed, it is advantageous to resolve the correlation by photographing the test bed plane in an oblique direction.

2. When $\kappa$ is $0^{\circ}$, the correlations of $x_{p}-Y_{0}$ and $y_{p}-X_{0}$ are close to 0 , and when $\kappa$ is $90^{\circ}$, the correlations of $x_{p}-X_{0}$ and $y_{p}-Y_{0}$ are close to 0 .

Next, the characteristics of the correlations between the orientation parameters identified through this paper are as follows.

3. Perspective and fish-eye lens projection cameras show different correlations depending on the type of test bed and the camera shooting method. It is relatively advantageous to use a V-type or plane-type test bed in an oblique direction to resolve the correlation between the orientation parameters of the fish-eye lens. On the other hand, in the A-type test bed, the correlation between the camera orientation parameters of the fish-eye lens is very high.

4. The correlation of $f-Z_{0}$ is not affected by $\kappa$, unlike the correlations of $x_{p}-X_{0}, y_{p}-Y_{0}, x_{p}-Y_{0}$, and $y_{p}-X_{0}$.

5. If the test bed, photographing angle, and number of object points are the same, the closer the distance between the camera and the test bed, the lower the correlation between the orientation parameters.

6. All the cases of correlation analysis dealt in this paper are not affected by focal length change. In other words, the above-mentioned correlation analyses (1-5) are valid for cameras with different focal lengths.

On the basis of the above-mentioned correlation analyses, the following factors should be considered in order to lower the correlation between the orientation parameters of the fish-eye lens camera.

- When all images with $\kappa=0$ and $90^{\circ}$ are used for self-calibration, the correlations of $x_{p}-X_{0}$, $y_{p}-Y_{0}, x_{p}-Y_{0}$, and $y_{p}-X_{0}$ can be eliminated at the same time (2).

- It is advantageous to use a V-type test bed to lower the correlation between the IOPs and EOPs of a fish-eye lens camera (1 and 3).

- The correlation of $f-Z_{0}$ cannot be adjusted through the setting of $\kappa$ (4). Therefore, to resolve the correlation of $f-Z_{0}$, the type of test bed and the relative position between the test bed and the camera must be handled with care $(1,3$, and 5$)$. 


\section{Conclusions}

This study was conducted to show the (i) analyses of correlation trends depending on the test bed type and the relative location of the test bed, and (ii) guidelines for acquiring reliable IOPs and EOPs of a camera.

It was confirmed that the correlation between the orientation parameters of each projection model varies according to the test bed type and the relative locations of the test bed and camera. The tendency of the correlation showed a large difference between the perspective projection and the fish-eye projection. In particular, the perspective projection showed the same level of correlation in the A- and V-type test beds, but the correlation of the fish-eye projection models showed a tendency to increase in the order of V, plane, and A types. In other words, the V-type test bed is advantageous in resolving the correlation of camera parameters. However, it does not effectively provide well-distributed points in the images to resolve camera lens distortion owing to the shape of the test bed.

In contrast, the A-type test bed provides a high correlation problem; however, a sufficient number of object points appeared and are well-distributed in the images. In this regard, a new type of test bed (e.g., a combination of V- and A-type test beds) designed to achieve reliable orientation and distortion parameters will be discussed in future work.

\section{Acknowledgments}

This work was supported by the National Research Foundation of Korea (NRF) grant funded by the Korea government (MSIT) (No. NRF-2019R1A2C1011014).

\section{References}

1 J. S. Sánchez, A. Gerhmann, P. Thevenon, P. Brocard, A. B. Afia, and O. Julien: Proc. 2016 Int. Tech. Meeting of the Institute of Navigation Conf. (ITM, 2016).

2 I. Marković, F. Chaumette, and I. Petrović: Proc. 2014 IEEE ICRA (IEEE, 2014) 5630.

3 D. Caruso, J. Engel, and D. Cremers: Proc. 2015 IEEE/RSJ Int. Conf. IROS (IEEE, 2015) 141

4 K. K. Sreedhar, A. Aminlou, M. M. Hannuksela, and M. Gabbouj: Proc. 2016 IEEE Int. Symp. Multimedia (ISM) (IEEE, 2016) 295.

5 T. Schöps, T. Sattler, C. Häne, and M. Pollefeys: Proc. 2015 Int. Conf. 3DV (IEEE, 2015) 291.

6 M. Campos, A. Tommaselli, E. Honkavaara, F. Prol, H. Kaartinen, A. El Issaoui, and T. Hakala: Sensors 18 (2018) 827. https://doi.org/10.3390/s18030827

7 C. Kühling: Fisheye Camera System Calibration for Automotive Applications Type (Freie Universität Berlin, Berlin, 2017).

8 C. Mei and P. Rives: Proc. 2007 IEEE ICRA (IEEE, 2007) 3945.

9 J. Marcato Junior, M. V. A. d. Moraes, and A. M. G. Tommaselli: Bol. Ciênc. Geod. 21 (2015) 637. http:// dx.doi.org/10.1590/S1982-21702015000300036

10 K. Miyamoto: J. Opt. Soc. Am. 54 (1964) 1060. https://doi.org/10.1364/JOSA.54.001060

11 C. Hughes, P. Denny, E. Jones, and M. Glavin: Appl. Opt. 49 (2010) 3338. https://doi.org/10.1364/AO.49.003338

12 S. Abraham and W. Förstner: ISPRS J. Photogramm. Remote Sens. 59 (2005) 278. https://doi.org/10.1016/ j.isprsjprs.2005.03.001

13 S. F. Ray: Applied Photographic Optics: Imaging Systems for Photography, Film, and Video (Focal Press, Oxford, UK, 2002)

14 J. Kannala and S. S. Brandt: IEEE Trans. Pattern Anal. Mach. Intell. 28 (2006) 1335. https://doi.org/10.1109/ TPAMI.2006.153 
15 S. Li: Proc. IEEE Conf. ICPR'06 (IEEE, 2006) 386.

16 C. Hughes, R. McFeely, P. Denny, M. Glavin, and E. Jones: IVC 28 (2010) 538. https://doi.org/10.1016/ j.imavis.2009.09.001

17 C. Sahin: J. Sens. 2016 (2016) 11. http://dx.doi.org/10.1155/2016/9379203

18 D. Schneider, E. Schwalbe, and H.-G. Maas: ISPRS J. Photogramm. Remote Sens. 64 (2009) 259. https://doi. org/10.1016/j.isprsjprs.2009.01.001

19 S. Urban, J. Leitloff, and S. Hinz: ISPRS J. Photogramm. Remote Sens. 108 (2015) 72. https://doi.org/10.1016/ j.isprsjprs.2015.06.005

20 H. A. Beyer: Geometric and Radiometric Analysis of a CCD-camera-based Photogrammetric Close-range System Type (ETH Zurich, Zurich, 1992).

21 K. B. Atkinson: Close Range Photogrammetry and Machine Vision (Whittles Publ., Dunbeath, UK, 1996).

22 K. H. Choi, Y. Kim, and C. Kim: Sensors 19 (2019) 1218. https://doi.org/10.3390/s19051218

23 D. C. Brown: Photogramm. Eng. 32 (1966) 444.

24 M. Li and J.-M. Lavest: IEEE Trans. Pattern Anal. Mach. Intell. 18 (1996) 1105. https://doi. org $/ 10.1109 / 34.544080$

\section{About the Authors}

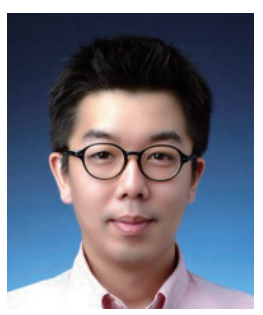

Kang Hyeok Choi received his B.S., M.S., and Ph.D. degrees from Seoul National University, South Korea, in 2011, 2013, and 2018, respectively. Since 2019, he has been a research professor at Myongji University. His research interests are in sensor system calibration and indoor and outdoor 3D reconstructions. (cwsurgy@naver.com)

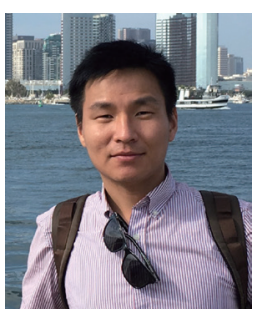

Yongmin Kim received his M.S. and Ph.D. degrees from Seoul National University, South Korea, in 2008, and 2012, respectively. From 2014 to 2017, he was a researcher at the National Disaster Management Research Institute, South Korea. Since 2019, he has been a researcher at LX, South Korea. His research interests are in monitoring urban environments.

(lovefortajo@gmail.com)

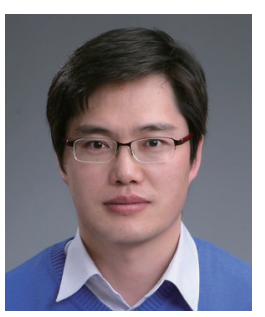

Changjae Kim received his B.S., and M.S. degrees from Seoul National University, South Korea, in 1998, and 2000, respectively. He received his Ph.D. degree from the University of Calgary, Canada, in 2009. Since 2014, he has been a professor at Myongji University. His research interests are in multisensor integration, building reconstruction, and indoor positioning. (cjkim@mju.ac.kr) 